\title{
Topic 1 Support Tools and Environments
}

\author{
Henryk Krawczyk, Jacques Chassin de Kergommeaux, \\ Pierre Manneback, and Tomás Margalef
}

Topic Chairs

Nowadays parallel distributed programmers use different tools and environments that facilitate the design, programming, testing, debugging and performance analysis and tuning of their applications. However, they do not satisfy all user requirements, such as broad usability, high effectiveness and proper accuracy. Therefore new propositions are still being developed and their properties tested on modern environments, such as clusters and grids. Their main aim is to simplify the understanding of what-and-why happens during execution of parallel and distributed applications. An important step is to prepare semantic descriptions of system behaviour and to make progress in high quality automatic analysis of performance bottlenecks.

This year 23 papers were submitted to this topic. Overall, they address different usability aspects of parallel distributed environments and tools to improve quality of program behaviour and performance analysis in such environments. The broad scope of considerations includes efficient distributed compilation, nested loop optimisation, checkpointing, system and software configuration management. Besides, monitoring, logging and tuning procedures design for different environments as well as middleware improvements to create high quality services are presented. Among the submissions, only seven papers (30\%) were finally accepted. They concentrate on improvements to the effectiveness and accuracy of the performance analysis of parallel and distributed programs. Novel approaches to these problems based on soft computing are presented. In particular, a high level query language is introduced to support performance analysis using linguistic expressions. Moreover, the performance profiling model is described to create a general algorithm for on-the-fly overhead assessment and compensation. The methods for improving performance of selected routine libraries are also discussed, and usability of analytical models and corresponding tools is also evaluated. New modelling techniques, based on occurrence and interrelationships of events, to build a data structure of a partial order of events is also given. Modification of existing middleware environments towards specification and dynamic adaptation of system services is also considered.

The qualified papers propose improvements in tools and parallel distributed environments and are a good material for foresting a discussion during session meetings. 